\title{
Tri-Logic: Um Ambiente Gamificado como Ferramenta de Auxílio ao ensino de aprendizagem de Lógica de Programação.
}

\author{
Maria Eduarda Contri Natal - IFRS Campus Canoas - dudacontri65@gmail.com \\ Brenda Anghinoni Barbosa - IFRS Campus Canoas - brendaab.2010@gmail.com \\ Júlia Cardoso Hernandes - IFRS Campus Canoas - juliachernandes@ gmail.com \\ Bruno de Sousa Much - IFRS Campus Canoas - muchsousa@gmail.com \\ Marcio Bigolin - IFRS Campus Canoas - marcio.bigolin@ canoas.ifrs.edu.br \\ Sandro José Ribeiro da Silva - IFRS Campus Canoas - sandro.silva@ canoas.ifrs.edu.br \\ Carla Balestro Silva - IFRS Campus Canoas - carla.silva@ canoas.ifrs.edu.br \\ Leonardo Filipe Batista de Carvalho - IFRS Campus Canoas - leonardo.carvalho@ canoas.ifrs.edu.br
}

Resumo: O presente trabalho propõe um ambiente gamificado, nomeado Tri-Logic, desenvolvido com o objetivo de servir de apoio ao ensino e aprendizagem na disciplina de lógica de programação. A partir de uma pesquisa bibliográfica, identificou-se que algumas das dificuldades encontradas no ensino e aprendizagem de habilidades de programação são as diversidades de ritmos dos alunos e sua falta de motivação. Uma das alternativas para enfrentar este problema é a utilização de técnicas da gamificação como estratégia, utilizando dinâmicas e mecânicas de jogos para motivar ações que não são, necessariamente, relacionadas a jogos. $\mathrm{O}$ ambiente desenvolvido, que vem sendo testado com os alunos do primeiro ano do curso técnico em Desenvolvimento de Sistemas integrado ao ensino médio, permitiu identificar resultados promissores, nos quais observa-se que a gamificação está diretamente ligada ao aumento da motivação dos alunos.

Palavras-chave: gamificação, ambientes virtuais de aprendizado, ensino de lógica de programação.

\section{Tri-Logic: A Gamified Environment as an Aid Tool for the Teaching and Learning of Programming Logic}

\begin{abstract}
The present paper proposes a gamified environment, named Tri-Logic, developed with the purpose to serve as a support in teaching and learning programming logic. From a bibliographic search, it was identified that some of the difficulties found in teaching and learning programming abilities are the diversity of students learning pace and their lack of motivation. One of the alternatives to face this problem is the use of gamification techniques as strategy, using dynamics and mechanics of games to motivate actions that are not necessarily related to games. The developed environment that has been tested with freshman students of Systems Development technical course integrated to high school, allowed the identification of promising results, in which is observed that gamification is directly connected with the rise of motivation among students.
\end{abstract}

Keywords: gamification, learning virtual environments, programming logic teaching.

\section{Introdução}

O desenvolvimento de habilidades de programação de sistemas computacionais vem sendo destacado como uma necessidade crescente, devido ao amplo uso de recursos 
computacionais nos mais diversos ramos. Ao mesmo tempo é conhecida a deficiência existente quanto à quantidade de profissionais sendo formados nesta área (Soares, 2016).

Um estudo realizado por Souza, Batista e Barbosa (2016) revelou que os principais elementos para a dificuldade dos estudantes no aprendizado de disciplinas de programação são a falta de motivação, junto com a dificuldade em compreender e aplicar os conceitos aprendidos. Uma proposta que vem sendo adotada para buscar uma forma de motivar os alunos, trazendo o ensino da programação para dentro de um ambiente que lhes é familiar, é o uso da gamificação. Para Paulo, Júnior e Boniati (2015), o ensino de programação pode ser apoiado pelo uso da técnica de gamificação, que consiste em utilizar conceitos, mecânicas e dinâmicas de jogos para motivar os alunos no desempenho de outras ações não relacionadas a jogos.

Gomes e Mendes (2016) ressaltam que uma grande barreira no aprendizado da programação está na incapacidade do aluno em conseguir acompanhar o ritmo de aula proposto pelos professores. Isso acontece porque na grande maioria das instituições de ensino, as aulas não são individuais, existindo uma ampla diversidade entre os alunos, seus ritmos e necessidades. O tema principal deste trabalho está associado com a exploração da gamificação como estratégia no ensino de lógica de programação no Curso Técnico em Desenvolvimento de Sistemas Integrado ao Ensino Médio. Foram analisados, em experimentos práticos por meio de oficinas, os potenciais do uso da gamificação como uma ferramenta efetiva no ensino e aprendizagem da lógica de programação, através do desenvolvimento e utilização de um ambiente gamificado denominado Tri-Logic. Para a validação do trabalho desenvolvido, foram realizados testes através de oficinas com alunos que possuem a disciplina de lógica de programação em seu currículo escolar e, após isso, solicitado que os mesmos respondessem a questionários de feedback. Os resultados obtidos foram positivos em sua maior parte e serão explorados na seção de Avaliações.

\section{Disciplina de Lógica de Programação}

A disciplina de lógica de programação é fundamental para a aprendizagem de qualquer linguagem de programação e acontece, geralmente, no primeiro semestre ou ano de diversos cursos na área de desenvolvimento de sistemas em Tecnologia da Informação (TI). É importante ressaltar que a apropriação ou não dos conceitos iniciais de programação tem relação direta com o desempenho dos alunos no decorrer de todo o curso, visto que as disciplinas mais avançadas dependem destes conceitos (Rocha, 2010).

Estudar lógica tende a melhorar a habilidade de pensar, de formular questões e aplicar resoluções a problemas cada vez mais difíceis. De maneira geral, a lógica consiste na arte de pensar corretamente (Friederich, 2012). Ela se relaciona com a programação de modo que seu estudo desenvolve um melhor raciocínio do profissional. Na programação é observado que é necessário um bom entedimento lógico para a realização das tarefas propostas (Tomazi, 2013). A disciplina de Lógica de Programação apresentada nesta seção implica em técnicas para melhorar e aprofundar o conhecimento de encadear pensamentos a fim de atingir um determinado objetivo dentro dos conceitos de programação (Nizzola, 2011).

É de fundamental importância que se aprimore o processo de ensino e aprendizagem da lógica de programação, pois um dos maiores gargalos nos cursos da área de TI é o alto índice de reprovação e/ou evasão nas disciplinas relacionadas à lógica cerca de 60\% - segundo Rocha et al. (2010). Nesse cenário, estão incluídos os alunos do 
primeiro ano do Curso Técnico em Desenvolvimento de Sistemas, no qual a média histórica dos últimos três anos é de 23,0 pontos percentuais, conforme observa-se na Tabela 1.

Tabela 1: Tabela de desempenho relacionado à disciplina de Lógica de Programação.

\begin{tabular}{|c|c|c|c|}
\hline Tabela & 2015 & 2016 & 2017 \\
\hline Aprovados & 26 & 27 & 30 \\
\hline Reprovados & 8 & 11 & 6 \\
\hline Infrequentes & 0 & 0 & 0 \\
\hline $\begin{array}{cc}\text { Taxa } & \text { de } \\
\text { aprovacão } & \end{array}$ & $76,5 \%$ & $71,1 \%$ & $83,3 \%$ \\
\hline $\begin{array}{ll}\text { Taxa } & \text { de } \\
\text { reprovação }\end{array}$ & $23,5 \%$ & $28,9 \%$ & $16,7 \%$ \\
\hline $\begin{array}{l}\quad \text { Média } \\
\text { Histórica } \\
\text { Reprovação }\end{array}$ & \multicolumn{3}{|c|}{$23,0 \%$} \\
\hline
\end{tabular}

Fonte: autoria própria

O problema mais preocupante relacionado ao ensino e aprendizagem de lógica de programação é a diversidade de ritmos de aprendizagem dos alunos, conjugada com grandes turmas e a falta de motivação. Assim, o aluno não percebe a relevância da atividade proposta e não se engaja na realização de uma atividade.

\section{Gamificação}

O termo "gamificação" foi primeiramente citado por um programador e inventor britânico chamado Nick Pelling, o qual tinha a ideia de que conceitos, mecânicas e dinâmicas dos games poderiam ser ampliados a contextos na realidade para engajar as pessoas a resolverem problemas. Porém, a gamificação ganhou forças e conhecimento somente a partir de 2010, quando âmbitos empresariais começaram a criar sistemas de recompensas e conquistas em seus softwares, visando um mercado favorável em que estas ideias seriam utilizadas para o crescimento da interação entre os serviços e os consumidores (Costa, 2014) (Alves, 2015).

Segundo Werbach e Hunter (2012), a gamificação pode ser dividida em três tópicos: interna, externa e para mudança de comportamento. No caso da gamificação interna, é tratado o uso de mecânicas e elementos de jogos em situações internas de empresas que visam o engajamento dos seus funcionários em prol de melhores resultados. A externa se refere também ao meio empresarial, onde o objetivo da gamificação é voltado para o envolvimento e crescimento de vendas a partir do cliente. Por outro lado, a gamificação para mudança de comportamento, que é a apresentada neste trabalho, envolve a mudança de hábitos ou atitudes de um indivíduo para que eles atinjam a meta proposta, seja no âmbito empresarial, educativo, da saúde, entre outros (Costa, 2014).

Elementos originários de games encontrados na gamificação também podem ser diferenciados em três, dinâmicas, mecânicas e os componentes (Alves, 2015). As dinâmicas são constituídas por elementos responsáveis por atribuir padrões regulares, sentido e coerência à experiência, ou seja, a estrutura e regras do game, e alguns outros elementos conceituais, como por exemplo, as emoções, a narrativa, a progressão, os relacionamentos e as constrições. As mecânicas são os elementos que movimentam toda 
a sistematização do game por parte do usuário, como os desafios, a cooperação e competição, o feedback, a aquisição de recursos, as recompensas, os estados de vitória, entre outros. Por fim, os componentes são definidos como as aplicações adicionais do jogo que implicam a formação do sistema de jogo, que são as realizações do usuário no jogo, os avatares, os badges, as coleções, o desbloqueio de conteúdos, os níveis, pontos, etc. (Alves, 2015). Estes conceitos serão necessários para o entendimento da seção 7.

Pode-se citar como exemplo do uso de gamificação (não necessariamente relacionado ao seu uso para ensino e aprendizagem) o aplicativo Nike + Running, no qual foi desenvolvido elementos de games como pontuação, progressão, desafios, relacionamentos, recompensas e pontuação, a fim de aumentar o incentivo direto ao usuário para que o mesmo pratique as atividades propostas no aplicativo, no caso, correr, caminhar, entre outras funções que visam proporcionar uma vida saudável ao cliente (Brunello, 2014). Na área da educação e aprendizado, podemos mencionar o aplicativo Duolingo, que visa o aprendizado de idiomas em práticas rápidas, simples e divertidas, tendo como estes idiomas, o Inglês, Espanhol, Francês e Alemão. Nele, são aplicados elementos como pontuação, recompensas, badges, níveis e feedback corretivo, que motivam o usuário a completar as tarefas propostas para o dia, e que, progressivamente, aumentam o nível do jogador, ampliando seus conhecimentos sobre o idioma escolhido.

Por fim, é possível concluir que o uso de metodologias ativas no processo de educação em suas diversas formas, tal como a gamificação, contribui para a inovação na sala de aula, deixando de lado a metodologia tradicional que vêm demonstrando muitas falhas no âmbito escolar, e ampliando a maneira como o conhecimento é adquirido pelo aluno, fazendo com que o mesmo se sinta motivado e realizado com o conhecimento aprendido. (Camargo; Daros, 2018).

\section{Análise de Softwares Relacionados}

Os softwares analisados estão resumidos na Tabela 2, de modo a permitir uma composição de aspectos e a identificação de motivadores para o desenvolvimento do trabalho proposto. Os softwares escolhidos foram Uri Online Judge, Scratch, Code Combat, Code Hunt e Codecademy. Na análise destes softwares foram observadas as suas diferenças e qualificações a fim de que estes possam ser aplicados no estudo do desenvolvimento do TriLogic.

Nos softwares analisados não estão presentes narrativas para transmitir informações e guiar os alunos, que permitiriam uma maior interatividade com o ambiente e motivandoos a superar seus desafios de uma forma lúdica e prazerosa (Battaiola; Dubiela, 2007). Outro ponto observado nos trabalhos analisados refere-se à falta de ludicidade. Em ambientes lúdicos, a carga de informação pode ser muito maior, os apelos sensoriais podem ocorrer em grande número e isso mantém o interesse do aluno e facilita a aprendizagem. 
Tabela 2: Tabela comparativa entre softwares relacionados.

\begin{tabular}{|c|c|c|c|c|c|}
\hline & URI & SaRATdA & CODECOMAT & chope & codeccademy \\
\hline $\begin{array}{l}\text { A que propõe } \\
\text { Ambiente (Ensino de } \\
\text { Programação)? }\end{array}$ & $\begin{array}{l}\text { C++, Java ou } \\
\text { Phyton }\end{array}$ & \begin{tabular}{l}
\multicolumn{1}{c}{ Conceitos } \\
Matemáticos e \\
Computacionais
\end{tabular} & $\begin{array}{l}\text { Python, } \\
\text { JavaScript, C++ } \\
\text { entre outras. }\end{array}$ & Java e C $\#$ & Java e PHP \\
\hline $\begin{array}{l}\text { Exige conhecimento } \\
\text { prévio de linguagens de } \\
\text { programação ou de } \\
\text { lógica? }\end{array}$ & Sim & Sim & Sim & Sim & Sim \\
\hline $\begin{array}{llr}\text { Informa } & \text { ao } & \text { usuário } \\
\text { quais } & \text { são } & \text { os } \\
\text { conhecimentos prévios? }\end{array}$ & Não & Não & Não & Sim & Não \\
\hline $\begin{array}{l}\text { Apresenta elementos } \\
\text { motivacionais? }\end{array}$ & Baixo & Baixo & Alto & Baixo & Baixo \\
\hline Ambiente é lúdico & Não & Não & Sim & Não & Não \\
\hline Possui narrativa? & Não & Não & Sim & Não & Não \\
\hline Possui tutorial online? & Não & Não & Não & Sim & Sim \\
\hline $\begin{array}{l}\text { Nivel de conhecimento } \\
\text { ao qual se destina }\end{array}$ & Intermediário & Intermediärio & Intermediário & Intermediário & Intermediário \\
\hline Tipo de ambiente & Web & Web & Web & Web & $\begin{array}{l}\text { Web e } \\
\text { Mobile }\end{array}$ \\
\hline
\end{tabular}

Fonte: Autoria Própria

\section{Metodologia}

Para o desenvolvimento do presente trabalho, optou-se pela utilização de uma abordagem qualitativa. Tal abordagem, segundo Gerhardt e Souza (2009), não está relacionada com a representatividade numérica, mas sim na compreensão e explicação da dinâmica das relações sociais, aspectos da realidade que não podem ser quantificados. Quanto à natureza, trata-se de uma pesquisa aplicada, correspondente ao conhecimento e uso de aplicações práticas voltadas à solução de problemas específicos. Este tipo de natureza, envolve verdades e interesses locais (Silva; Menezes, 2005).

No primeiro momento, foi realizada uma ampla pesquisa bibliográfica e também o estudo em grupo de trabalho com foco em educação e gamificação. Após isso, foram identificados os requisitos necessários para um ambiente gamificado de ensino e aprendizagem de lógica de programação e desenvolvido um protótipo que possibilitou testes práticos realizados através de oficinas com os alunos.

Os resultados obtidos foram avaliados com base nas premissas levantadas na fase de requisitos e possibilitaram modificações no ambiente que consiste na atual versão do ambiente. Esta versão também passará por uma etapa de testes a fim de validar sua aceitação e eficiência entre os estudantes.

\section{Desenvolvimento do Ambiente Gamificado}

$\mathrm{Na}$ fase de concepção do ambiente gamificado Tri-Logic foram levantados os requisitos necessários para que o mesmo permitisse atender os objetivos de ensinar lógica de programação, levantados com professores da instituição com base nos resultados de avaliações da disciplina de em anos anteriores, através de conceitos básicos com o auxílio de atividades lúdicas, motivando o aluno a continuar jogando até completar todos os níveis. Através deste levantamento, observou-se que o ambiente deveria contar com as seguintes características: a) temática condizente com a faixa etária do público alvo; b) cada tema deveria ser abordado em dois ou mais níveis; c) nível de dificuldade crescente; d) menus de navegação simples e intuitivos; e e) jogabilidade simplificada, a fim de não desencorajar alunos não acostumados com ambientes gamificados. Como estudo de caso, 
foram destinadas atividades para alunos iniciantes do Curso Técnico em Desenvolvimento de Sistemas, que permitiam uma visão geral sobre a construção da lógica de programação através de seus conceitos básicos.

Após o levantamento dos requisitos, iniciou-se a fase de modelagem (Figura 1), diagramação e implementação do ambiente. Como mostrado na Figura 1, o aluno acessa o ambiente através de seu dispositivo e é redirecionado para o Tri-Logic, juntamente com esse redirecionamento alguns dados do aluno serão salvos, tais como nome e ID. A partir disso, toda vez que o aluno entrar em um nível e realizar uma ação estes dados ficarão armazenados.

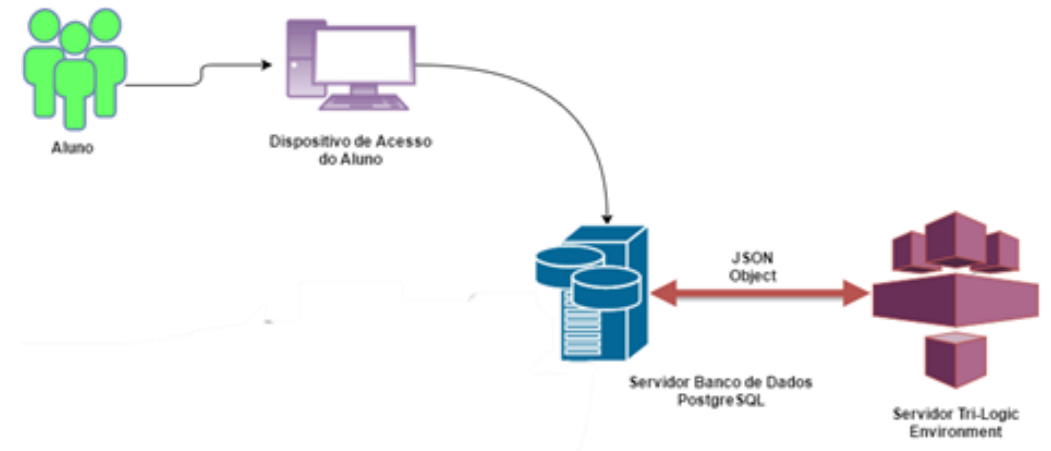

Figura 1: Modelo computacional do ambiente.

Os cenários das missões consistem em três visões: a apresentação da missão, o jogo e a modal de encerramento. Uma missão consiste em um conjunto de tarefas a serem executadas de modo semelhante no jogo, apropriando-se de um conceito previamente determinado relacionado ao objetivo principal do ambiente. Por outro lado, a apresentação da missão e a modal de encerramento são caracterizadas por uma tela prévia ou posterior do jogo que sinaliza sobre o conteúdo apresentado naquela missão. Primeiramente, a modal inicial demonstra uma definição prévia do conceito, e a modal de encerramento mostra a conclusão da missão com a utilização daquele conteúdo.

Utilizando-se do framework AngularJS, o cenário é montado empregando o directive, conceito que permite o reuso de componentes aplicados em todos os objetos da cena.

$\mathrm{O}$ arquivo CSS (folha de estilos) game-objects.css contém os diversos estilos que são aplicados aos objetos do jogo. A interatividade da cena é definida por um arquivo JavaScript, que, utilizando-se das estruturas do framework, monta o componente Controller da cena. Cada missão possui um objeto JSON que armazena os textos da cena, este objeto é carregado via o Service do framework, utilizando-se de requisições ajax . As requisições informam o ID da fase em questão e a linguagem do navegador do usuário. Esta prática facilita a tradução e a customização dos textos do jogo.

Ao acessar o ambiente o aluno será apresentado ao mesmo pela sua agente animada Brenda (Figura 3), que o sinalizará sobre as fases e a mecânica do jogo. A opção pelo agente pedagógico tem base nos trabalhos de Reategui et al. (2006), onde acredita-se que a implementação de um agente pedagógico animado desempenhando o papel de um assistente integrado ao ambiente possa contribuir para a melhoria do processo de ensinoaprendizagem dos alunos que o utilizarem. Além de incentivar, o agente pedagógico animado também diminui as chances de comportamentos inadequados do aluno, tais como o Gaming the System , (a trapaça)(Nunes; Jaques, 2013). 


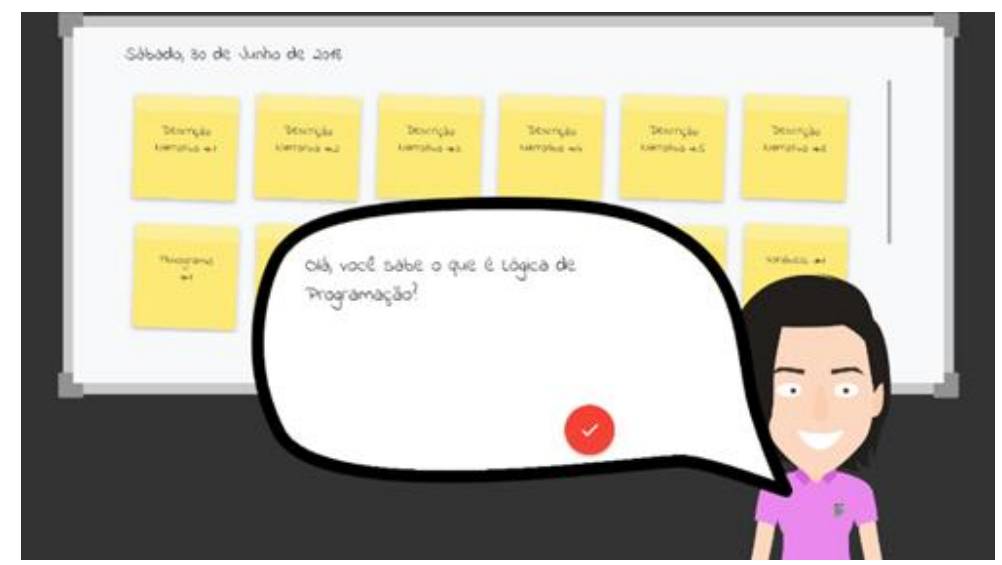

Figura 3: Tela de apresentação do ambiente.

A tela apresentada na Figura 4 apresenta os elementos envolvidos na narrativa do ambiente, compostos por objetos de ação e objetos alvos durante a execução dos níveis. Nesta demonstração, a fase de Descrição Narrativa está finalizada e a agente Brenda apresenta o feedback.

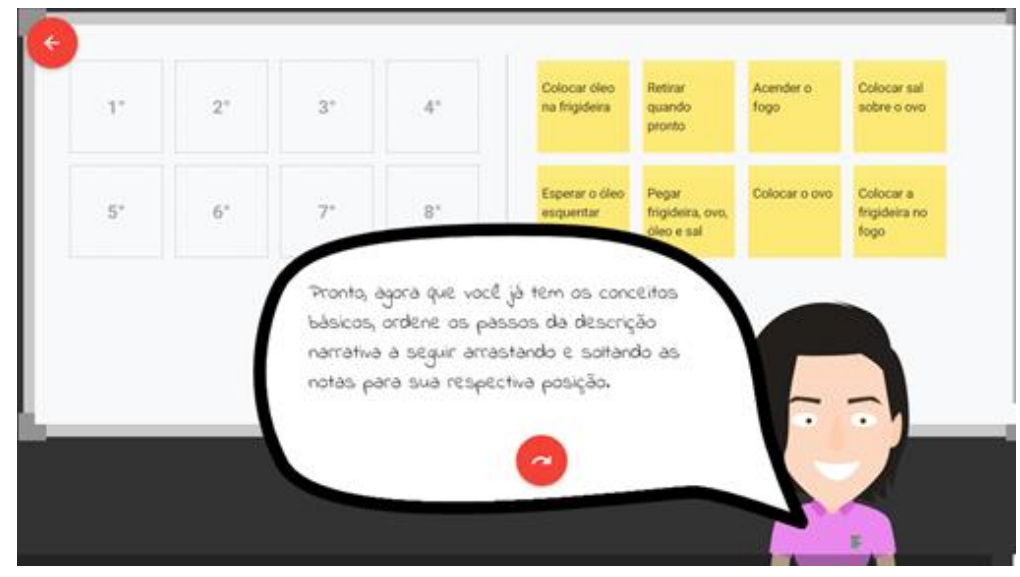

Figura 4: Tela da fase de Descrição Narrativa.

A utilização da opção de arrastar-e-soltar como mecânica se deu pela fácil visualização das respostas por parte do aluno, assim, ele pode visualizar a execução das suas respostas como na estruturação de um programa (Rino, 2018). Nessas fases, estes objetos completam a missão sendo arrastados às suas devidas lacunas.

A Modal de Encerramento finaliza a missão, apresentando ao jogador uma descrição da fase executada e uma retomada do conteúdo visto. De acordo com a fase há, também, uma representação da atividade realizada utilizando pseudocódigo, conforme apresentado na modal da Figura 7. 


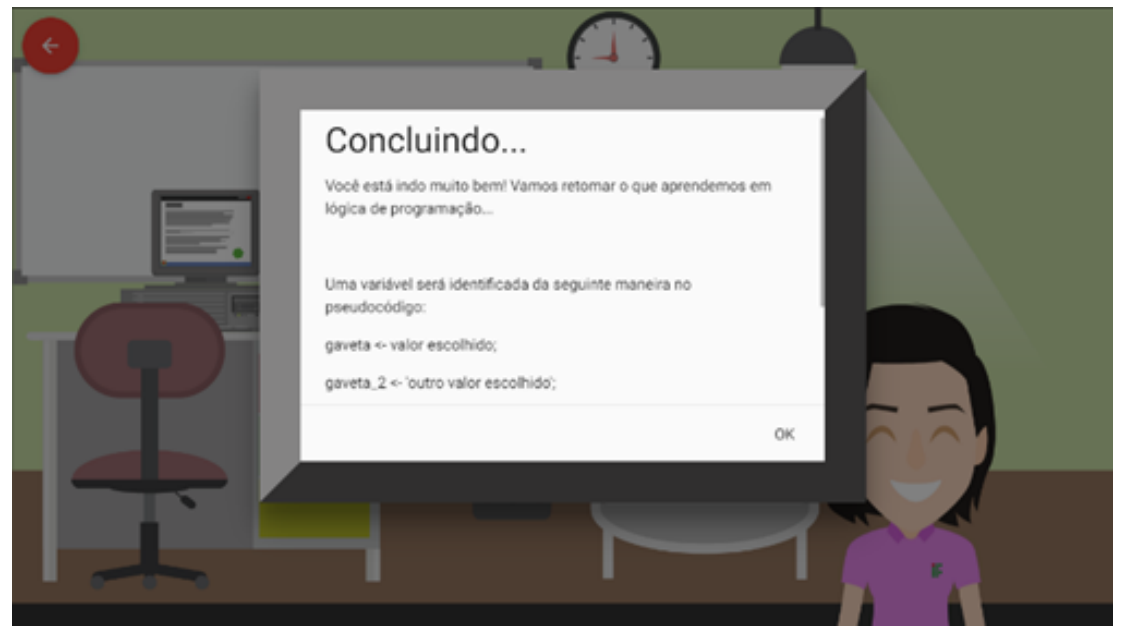

Figura 7: Tela de encerramento da fase.

\section{Avaliações}

No presente ano, a primeira atividade foi uma oficina introdutória, na qual o projeto é apresentado aos participantes, de forma com que eles possam se familiarizar com os conceitos e termos utilizados e compreender o objetivo da atividade. Contando com a participação de sete alunos, durante uma hora, foram propostos a eles jogos que estimulassem o raciocínio lógico. Posteriormente, foi realizada a segunda oficina, que iniciou a fase de utilização do ambiente e contou com a participação de mais alunos.

Diferente das atividades anteriores, as oficinas utilizando o Tri-Logic vem sendo realizadas em etapas, devido ao desenvolvimento de novas fases no ambiente. Desta forma, os conteúdos são mais explorados individualmente, a fim de reforçar a aprendizagem do aluno, e trabalhados gradativamente conforme o plano de ensino da disciplina. Nesta primeira etapa, foram disponibilizadas 13 fases que abrangiam três conceitos distintos, sendo eles descrição narrativa, fluxograma e variáveis.

Durante uma hora, os 15 alunos participantes puderam realizar a atividade, trocando ideias com colegas ou tirando dúvidas com os monitores presentes. Ao fim do período e após terem finalizado todas as fases, foi solicitado que respondessem a um questionário de feedback. O questionário aplicado foi reformulado com questões que pudessem avaliar quesitos como motivação, aprendizagem e aceitação do ambiente através da percepção do usuário. Analisando aspectos de aprendizagem, 67,7\% dos participantes disseram que o ambiente Tri-Logic contribuiu bastante ou razoavelmente para sua aprendizagem na disciplina. Todos os participantes acreditam que o uso de ambientes gamificados pode auxiliar no aprendizado de disciplinas em geral. No quesito de motivação, os resultados também foram positivos em sua maioria. Sobre quão divertida foi a utilização do ambiente, $73,3 \%$ afirmaram ter sido divertida e 26,7\% muito divertida. Em relação ao feedback realizado pelo Agente pedagógico, 53,3\% dos alunos afirmaram que este fator os motivou a continuar. Dos participantes, $60 \%$ sentiam-se confiantes em passar em um teste sobre os conteúdos abordados após a utilização do ambiente, 6,7\% não souberam responder e o restante disse sentir-se mais ou menos ou nada confiante. Em "Você sentiuse motivado a aprender sobre lógica de programação?", $88,7 \%$ dos alunos responderam sim. 
Ao final do questionário, foram deixadas algumas sugestões e comentários sobre a experiência. Entre as respostas, destacaram-se propostas para que as fases possuam níveis de dificuldade maior e o uso de recompensas. Todas as sugestões serão colhidas e analisadas, a fim de serem utilizadas na otimização do ambiente.

\section{Conclusões}

O Tri-Logic passou por períodos de testes, que apresentou resultados positivos da implementação da gamificação no ambiente acadêmico. Os resultados obtidos possibilitaram a aplicação de melhorias, dentre elas a reformulação das fases iniciais. Porém, há de ser observado que os pontos negativos indicados nos testes não impediram o ambiente de atingir seu objetivo, uma vez, que os alunos relataram que tiveram uma experiência gamificada agradável e que conseguiram aprender o conteúdo proposto através do ambiente. Na Tabela 1 vista anteriormente, é possível observar que do ano de 2016 para 2017 houve uma considerável queda na quantidade de alunos reprovados. Diante disso, é importante ressaltar que durante esse período o projeto já estava em andamento e o ambiente estava sendo aplicado aos alunos, levando a crer que o uso do Tri-Logic, tal como a aplicação de técnicas da gamificação na educação, possa ter melhorado o desempenho dos estudantes na disciplina.

De acordo com os resultados preliminares obtidos do questionário aplicado no ano corrente, podemos concluir que o ambiente Tri-Logic conseguiu desenvolver uma construção da motivação do estudante ao ser utilizado, tendo em vista que a maioria dos alunos $(88,7 \%)$ se sentiram motivados a ampliar seus conhecimentos na disciplina de Lógica de Programação após a realização das oficinas.

\section{Referências}

BATTAIOLA, A.; DUBIELA, R.. A Importância das Narrativas em Jogos de Computador. VI Simpósio Brasileiro de Jogos para Computador e Entretenimento Digital (SBGAMES), Novo Hamburgo/RS, 2007.

COSTA, Luis Antonio. A "Gamificação" e o poder dos games na transformação da sociedade. 2014. Disponível em: <http://www.gameblast.com.br/2014/07/agamificacao-e-o-poder-dos-games-na.html>. Acesso em: 12 dez. 2017.

FRIEDERICH, R. et al. Proposta Metodologica para a Inserção ao ao Ensino de Lógica de Programação com Logo e Lego Mindstorms. XXIII Simpósio Brasileiro de Informática na Educação (SBIE), Rio de Janeiro/RJ, 2012.

GERHARDT, Tatiana Engel; SOUZA, Aline Corrêa de. Métodos de Pesquisa. Porto Alegre: Editora da Ufrgs, 2009. 120 p. Disponível em: <http://www.ufrgs.br/cursopgdr/downloadsSerie/derad005.pdf>. Acesso em: 12 jun 2018.

GOMES, A.; MENDES, A. J. N. Learning to program - difficulties and solutions. ICEE - International Conference on Engineering Education. Anais...Coimbra: International Conference on Engineering Education, 2007. Disponível em: <http://icee2007.dei.uc.pt/proceedings/papers/411.pdf>. Acesso em: 22 maio 2016. 
GUIMARÃES, S. E. R. Motivação intrínseca, extrínseca e o uso de recompensas em sala de aula. In: BZUNECK, J.A.; BORUCHOVITCH, E. (Orgs.). A Motivação do aluno: contribuições da psicologia contemporânea. Petrópolis: Vozes, 2009. p. 37-57.3

NIZZOLA, Márcio Rogério. Introdução Sobre Lógica. 2011. Disponível em: < http://nizzola.com.br/L\%C3\%B3gica_de_Programa\%C3\%A7\%C3\%

A3o,Aulas_de_L\%C3\%B3gica,Exerc\%C3\%ADcios_de_L\%C3\%B3 gica,Programa\%C3\%A7\%C3\%A3o/12/Introdu\%C3\%A7\%C3\%A3o _sobre_L\%C3\%B3gica>. Acesso em: 28 jul. 2018.

NUNES, Thiago Marquez; JAQUES, Patrícia A.. Analisando a influência da presença de um Agente Pedagógico Animado em relação ao Gaming The System. XXIV Simpósio Brasileiro de Informática na Educação (SBIE), Campinas/SP, 2013.

PAULO, R.; JÚNIOR, M.; BONIATI, B. B. LogicBlocks : Uma Ferramenta para o Ensino de Lógica de Programação in EATI - Encontro Anual de Tecnologia da Informação e Semana Acadêmica de Tecnologia da Informação, Frederico Westphalen, 2015.

REATEgui, E. B. et al. Agentes Pedagógicos Animados. Renote Revista Novas Tecnologias na Educação, v. 4, n. 2, p. 1-10, 2006.

RINO, Marcelo Valério. Estratégias para a Utilização de Jogos Digitais e Tecnologias Emergentes no Ensino De Linguagem De Programação. Dissertação (Mestrado em Mídia e Tecnologia )- Universidade Estadual Paulista "Júlio de Mesquita Filho". Faculdade de Arquitetura, Artes e Comunicação, Bauru. 2018. Disponível em: < https://repositorio.unesp.br/handle/11449/154090>. Acesso em: 28 jul. 2018.

ROCHA, P. S. et al. Ensino e Aprendizagem de Programação: Análise da Aplicação de Proposta Metodológica Baseada no Sistema Personalizado de Ensino. RENOTE Revista Novas Tecnologias na Educação, v. 8, n. 3, p. 1-11, 2010.

SILVA, Edna Lúcia da; MENEZES, Estera Muszkat. Metodologia da Pesquisa e Elaboração de Dissertação. 4. ed. Florianópolis: Ufsc, 2005. 139 p. (1123). Disponível em: . Acesso em: 12 jun 2018.

SOARES, Nana. Crise? Não no mercado de Tecnologia da Informação. Estadão. 29 mar. 2016. Disponível em: <https://educacao.estadao.com.br/noticias/geral,crise-naono-mercado-de-tecnologia-da-informacao,10000023666>. Acesso em: 3 jun. 2016.

SOUZA, Draylson Micael; BATISTA, Marisa Helena da Silva; BARBOSA, Ellen Francine. Problemas e Dificuldades no Ensino e na Aprendizagem de Programação: Um Mapeamento Sistemático. Revista Brasileira de Informática na Educação, 2016. 2

TOMAZI, Rodrigo Angelo. Lógica: A Arte de Pensar. Pato Branco - Pr: [s.n], [2013]. 11 p. Disponível em: <https://pt.scribd.com/document/61444933/Logica-A-Arte-dePensar>. Acesso em: 17 jul. 2018.

WERBACH, K.; HUNTER, D. For the Win: How Game Thinking Can Revolutionize Your Business. [s.1.] Wharton Digital Press, 2012.

CAMARGO, Fauto; DAROS, Thuinie. A Sala de Aula Inovadora: estratégias pedagógicas para fomentar o aprendizado ativo. Porto Alegre: Penso, 2018. 144 p. 